\title{
Model - Based Approach to Estimate dFRC in the ICU Using Measured Lung Dynamics
}

\author{
A.N. Mishra*, Y.S. Chiew*, J.G. Chase*, G.M. Shaw** \\ *University of Canterbury, Christchurch, 8041, New Zealand (e-mail: ankit.mishra@pg.canterbury.ac.nz, \\ yeong.chiew@pg.canterbury.ac.nz,geoff.chase@canterbury.ac.nz) \\ **Christchurch Hospital, Christchurch, 8011, New Zealand
}

\begin{abstract}
Acute Respiratory Distress Syndrome (ARDS) is characterized by inflammation and filling of the lung with fluid. Mechanical ventilation (MV) is used to treat ARDS/ALI using positive end expiratory pressure (PEEP) to recruit and retain lung units, thus increasing pulmonary volume and dynamic functional residual capacity (dFRC) at the end of expiration. However, simple methods to measure dFRC at the bedside currently do not exist and other methods are too invasive and impractical to carry out on a regular basis.

Stress-strain theory is used to estimate $\triangle \mathrm{dFRC}$, which represents the extra pulmonary volume due to PEEP, utilizing readily available patient data from a single breath. The model uses commonly controlled or measured parameters (lung compliance, plateau airway pressure, PV data) to identify a parameter $\boldsymbol{\beta}_{\mathbf{1}}$ as a function of PEEP and tidal volume. A median $\boldsymbol{\beta}_{\mathbf{1}}$ value is calculated for each PEEP level over a cohort and is hypothesised as a constant throughout the population for the particular PEEP. Estimated $\triangle \mathrm{dFRC}$ values are then compared to measured values to assess accuracy of the model.

$\triangle \mathrm{dFRC}$ was calculated for 9 patients and compared to the measured values. The median percentage error was 40.29\% (IQR = 14.20, 55.39) for PEEP $=5 \mathrm{cmH}_{2} \mathrm{O}, 31.12 \%(\mathrm{IQR}=10.53,192.71)$ for $\mathrm{PEEP}=10$ $\mathrm{cmH}_{2} \mathrm{O}, 20.8 \%(\mathrm{IQR}=7.51,81.06)$ for $\mathrm{PEEP}=15 \mathrm{cmH}_{2} \mathrm{O}, 15.44 \%(\mathrm{IQR}=11.92,36.18)$ for PEEP $=20$ $\mathrm{cmH}_{2} \mathrm{O}, 19.7 \%(\mathrm{IQR}=4.79,20.76)$ for PEEP $=25 \mathrm{cmH}_{2} \mathrm{O}$ and 11.78\% $(\mathrm{IQR}=2.99$, 27.5) for PEEP = $30 \mathrm{cmH}_{2} \mathrm{O}$. Linear regression between estimated and measured $\Delta \mathrm{dFRC}$ produced $\mathrm{R}^{2}=0.862$.

The model-based approach offers a simple and non-invasive method which does not require interruption of MV to estimate dFRC. The clinical accuracy of the model is limited but was able to track the impact of changes in PEEP and tidal volume on dFRC, on a breath-by-breath basis for each PEEP.
\end{abstract}

Keywords: Functional Residual Capacity, dFRC, FRC, Pulmonary, Model, ARDS, Intensive Care, ICU

\section{INTRODUCTION}

Acute Respiratory Distress Syndrome/Acute Lung Injury (ARDS/ALI) are characterized by inflammation and fluid filled lungs. Severely affected lung units collapse and cannot be recruited without external intervention, resulting in a smaller, stiffer lung, called the "baby lung" (Gattinoni and Pesenti, 2005). Mortality rates for ARDS have been reported between 30\% - 70\% (Bersten et al., 2002, Luhr et al., 1999, Manzano et al., 2005, Suchyta et al., 1997).

Clinicians offer a supportive environment that aids patient recovery by application of mechanical ventilation (MV), which partially or completely takes over the patients breathing effort. The primary focus of $\mathrm{MV}$ is to improve recruitment of lung units and increase gas exchange, while minimizing further harm (Ware and Matthay, 2000), primarily through application of a suitable positive end expiratory pressure (PEEP).

PEEP is important since ARDS affected lung units are vulnerable to collapse due to the extra pressure of the fluid and denaturing of surfactant that maintain the shape of the alveoli. PEEP prevents alveolar collapse and enhances gas exchange using higher pressure to recruit the collapsed alveoli. However, high PEEP and tidal volume $\left(\mathrm{V}_{\mathrm{t}}\right)$ can also damage healthy alveoli (Bersten, 1998).

Functional Residual Capacity (FRC) is the lung volume at zero end expiratory pressure (ZEEP). PEEP ensures pulmonary volume above FRC. This additional volume, dynamic FRC (dFRC) is schematically shown in Figure 1:

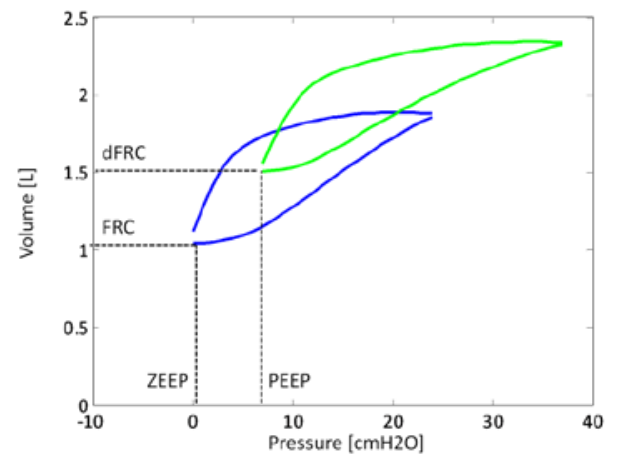

Fig. 1. dFRC at PEEP $>0$ and FRC at ZEEP 
dFRC offers useful clinical information regarding the lung recruitability, but is not normally measured at the bedside. Some mechanical ventilators allow FRC and dFRC measurements (GE Healthcare., 2006), but most do not. However, these measurements require interruption of $\mathrm{MV}$ and can be additionally invasive. Computer Tomography (CT) scans timed at the end of expiration allow accurate assessment of FRC (Malbouisson et al., 2001). However, it has limited application since it requires transport of the patient out of the Intensive care unit (ICU), disconnecting the patient from the ventilator and exposure to radiation. Other methods include washout of tracer gases, such as Nitrogen (Heinze et al., 2007, Olegard et al., 2005), which allows precise measurement of FRC, but requires the use of a dispensing device for the tracer gas, negating its frequent use at the bedside (Weismann et al., 2006).

dFRC is clinically important, can indicate lung condition, and, if tracked regularly, can identify changes in patient condition. The model proposed in this research aims to estimate dFRC from readily available pressure-volume (PV) data without interrupting MV treatment. The model is an extension to a previous model proposed by Sundaresan et al (Sundaresan et al., 2011) which required PV data at a minimum of two PEEP levels. More PV loops at a wide range of PEEP levels were required for higher accuracy. The new model proposed here requires PV data from only one breath at one PEEP level to estimate dFRC at different PEEP levels for a given patient. This major extension allows continuous tracking of dFRC at the patient's bedside without interrupting MV.

\section{METHODS}

\subsubsection{Model Summary}

Chiumello et al (Chiumello et al., 2008) proposed a stressstrain theory of lung dynamics which was used by Sundaresan et al (Sundaresan et al., 2011) to develop a model to estimate dFRC. Chiumello et al defined transpulmonary pressure $\left(\Delta \mathrm{P}_{\mathrm{L}}\right)$ as the clinical equivalent of stress. Transpulmonary pressure is the difference between the applied airway pressure and the corresponding pleural pressure. The clinical equivalent of strain is the ratio of the change in volume $(\Delta \mathrm{V})$ to the FRC, which represents the resting lung volume, yielding a stress-strain definition:

$$
\Delta \mathrm{P}_{\mathrm{L}}=\mathrm{E}_{\text {Lspec }} \times \frac{\Delta \mathrm{V}}{\mathrm{FRC}}
$$

Where, the specific lung elastance $\left(\mathrm{E}_{\mathrm{Lspec}}\right)$ can be defined as the transpulmonary pressure at which FRC effectively doubles. The values of specific lung elastance reported by Chuimello et al (Chiumello et al., 2008) were 13.4 \pm 3.4 $\mathrm{cmH}_{2} \mathrm{O}$ for the control subjects, $12.6 \pm 3.0 \mathrm{cmH}_{2} \mathrm{O}$ for the medical control group, $14.4 \pm 3.6 \mathrm{cmH}_{2} \mathrm{O}$ for the ALI subgroup, and $13.6 \pm 4.1 \mathrm{cmH}_{2} \mathrm{O}$ for the ARDS subgroup. The study indicated that specific lung elastance does not vary significantly across subgroups.

Transpulmonary pressure can also be defined in terms of plateau airway pressure $\Delta \mathrm{P}_{\mathrm{aw}}$ :

$$
\Delta \mathrm{P}_{\mathrm{L}}=\Delta \mathrm{P}_{\mathrm{aw}} \times \alpha
$$

Where:

$$
\propto=\frac{E_{L}}{E_{L}+E_{C W}}
$$

Where $\alpha$ in Equation 3 represents the static lung elastance, defined by the Lung Elastance $\left(\mathrm{E}_{\mathrm{L}}\right)$ and the Chest Wall Elastance $\left(\mathrm{E}_{\mathrm{CW}}\right)$. Chest wall elastance plays an important role in $\mathrm{MV}$ as part of the airway pressure applied is used to inflate the lungs and the rest is utilized to overcome chest wall pressure. This relation and the effect of chest wall elastance is shown in Figure 2.

It can be observed that the total elastance is the same in both cases in Figure 2. Case (a) is typical of ARDS patients where a stiffer lung is represented by the higher lung elastance compared to case (b), which shows a healthy lung. Hence, the value of $\alpha$ indicates the severity of ALI or ARDS, where a higher value of $\alpha$ indicates a higher severity of ARDS.

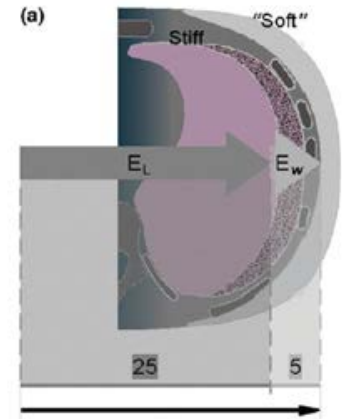

$E_{\text {tot }}$
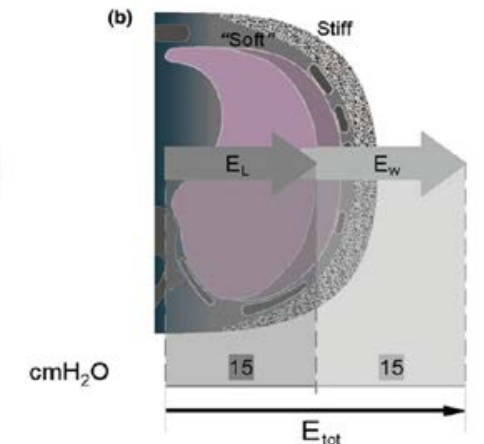

Fig. 2. Effects of different lung and chest wall elastance (Gattinoni et al., 2004)

The values of $\alpha$ reported by Chiumello et al (Chiumello et al., 2008) were $0.69 \pm 0.15$ for control group patients, $0.74 \pm 0.16$ for intensive care patients with medical diseases, $0.64 \pm 0.15$ for the ALI subgroup and 0.71 \pm 0.16 for ARDS subgroup.

Combining Equations 1 and 2 yields:

$$
\mathrm{FRC}=\frac{\Delta \mathrm{V}}{\Delta \mathrm{P}_{\mathrm{aw}}} \times \frac{\mathrm{E}_{\mathrm{Lspec}}}{\propto}
$$

Equation 4 defines FRC as a function of the lung compliance $\left(\frac{\Delta \mathrm{V}}{\Delta \mathrm{P}_{\mathrm{aw}}}\right), \mathrm{E}_{\mathrm{Lspec}}$ and $\alpha$ of the patient.

It was hypothesised that dFRC follows similar mathematical form as Equation 5. It should be noted that $\triangle \mathrm{dFRC}$ in Equation 6 represents the additional volume in the lung above FRC due to application of PEEP and not the absolute dFRC, which consists of the FRC as well.

$$
\mathrm{FRC}+\Delta \mathrm{dFRC}=\frac{\Delta \mathrm{V}}{\Delta \mathrm{P}_{\mathrm{aw}}} \times \frac{\mathrm{E}_{\mathrm{Lspec}}}{\propto}(1+\mathrm{x})
$$

Defining $\mathrm{dFRC}=\mathrm{FRC}+\Delta \mathrm{dFRC}$ yields:

$$
\Delta \mathrm{dFRC}=\frac{\Delta \mathrm{V}}{\Delta \mathrm{P}_{\mathrm{aw}}} \times \frac{\mathrm{E}_{\mathrm{Lspec}}}{\propto} \mathrm{X}
$$

Where $\mathrm{x}$ is a function of the PEEP level at which $\triangle \mathrm{dFRC}$ is estimated. $\mathrm{E}_{\mathrm{Lspec}}$ and $\alpha$ are constant parameters and can be combined into one parameter, $\beta$, yielding: 


$$
\Delta \mathrm{dFRC}=\frac{\mathrm{v}_{\mathrm{t}}}{\Delta \mathrm{P}_{\mathrm{aw}}} \times \beta
$$

Where $\Delta V$ is replaced by tidal volume $\left(V_{t}\right)$ and $\beta$ is a function of the PEEP applied, $\mathrm{E}_{\mathrm{Lspec}}$ and $\alpha$. The assumption that $\alpha$ is constant is true only for the linear portion of the PV loop (Sundaresan et al., 2011).

Sundaresan et al (Sundaresan et al., 2011) proposed a model to estimate the $\triangle \mathrm{dFRC}$ defined:

$$
\mathrm{dFRC}=\frac{\Delta \mathrm{dFRC}}{\Delta \mathrm{PEEP}} \times \beta
$$

Where $\frac{\Delta \mathrm{dFRC}}{\triangle \mathrm{PEEP}}$ represents the volume responsiveness of the lung to a change in PEEP and $\triangle \mathrm{dFRC}$ represents the additional volume due to PEEP. Sundaresan et al (Sundaresan et al., 2011) also hypothesized that the value of $\beta$ for a particular level of PEEP can be assumed as a population constant for a given PEEP, which is used here.

\subsubsection{Patients}

Clinical data for 9 patients (Sunderesan, 2010) in Table 1. The data is characterized by different levels of lung injury, including PV data for at least 3 PEEP levels per patient. The data did not contain information on the FRC of the lung but contained the measured $\triangle \mathrm{dFRC}$ values for each PEEP level for each patient. This study uses an average breath for each PEEP level. The use of data was approved by the New Zealand South Island Regional Ethics Committee.

Table 1: Characteristics of the patient studied

\begin{tabular}{|c|c|c|c|}
\cline { 2 - 4 } & Sex & Age [years] & Cause of lung injury \\
\hline \hline Patient 1 & Female & 61 & Peritonitis \\
Patient 2 & Male & 22 & Trauma \\
Patient 3 & Male & 55 & Aspiration \\
Patient 4 & Male & 88 & Pneumonia \\
Patient 5 & Male & 59 & Pneumonia \\
Patient 6 & Male & 69 & Trauma \\
Patient 7 & Male & 56 & Legionnaires \\
Patient 8 & Female & 45 & Aspiration \\
Patient 9 & Male & & H1N1 \\
\hline
\end{tabular}

\subsubsection{Analyses \& New Approach}

The proposed model estimates $\triangle \mathrm{dFRC}$ based on the compliance $\left(\frac{\mathrm{V}_{\mathrm{t}}}{\Delta \mathrm{P}_{\mathrm{aw}}}\right)$ observed in the PV data recorded for each patient. A representative breath, with PEEP level and corresponding peak inspiratory pressures normally observed in clinical settings was selected to calculate the lung compliance for each patient since the lung compliance was found to change with pressure. In particular, it was found to decrease at higher PEEP levels. Since the FRC values were not measured , $\frac{E_{\text {Lspec }}}{\alpha}$ could not be estimated. Instead, the $\beta$ was calculated using Equation 8. Calculated $\beta$ values were then normalized by tidal volume $\left(V_{t}\right)$ as $d F R C$ can vary with the $V_{t}$ applied, yielding:

$$
\beta_{1}=\frac{\beta}{\mathrm{v}_{\mathrm{t}}}
$$

A median $\beta_{1}$ value was calculated for each PEEP level over all patients, which was used as a population constant. The $\triangle \mathrm{dFRC}$ value for each PEEP level for each patient was then estimated by substituting this median $\beta_{1}$ value into Equation 7 , and multiplying the resultant value with the applied $V_{t}$. The median and (IQR) of errors are reported for all $(\mathrm{N}=53)$ data points (patients and PEEP levels).

The linear trend observed in median $\beta_{1}$ values at each PEEP level over the cohort used to linearly interpolate median $\beta_{1}$ values at intermediate PEEP levels to generalise the approach. These interpoloated $\beta_{1}$ values were then used to reestimate $\triangle \mathrm{dFRC}$ values at all the PEEP levels, using only surrounding data to interpolate from.

Overall, this new approach and analysis can be summarised:

- Calculate $\beta_{1}$ for each patient and PEEP

- Use median $\beta_{1}$ for each PEEP as a population constant to estimate $\triangle \mathrm{dFRC}$ at those PEEP values for each patient

- Assess error between calculated and measured $\triangle$ dFRC values.

This approach requires values for $\beta_{1}$ from patient data at all PEEP values that might be used. To generalise this approach, interpolating $\beta_{1}$ between known $\beta_{1}$ values at different PEEP levels was tested. Thus:

- $\quad$ Using median cohort $\beta_{1}$ values for two surrounding or near PEEP values, an interpolated $\beta_{1}$ is calculated for each PEEP level.

- Interpolated $\beta_{1}$ for each PEEP as a population constant to estimate $\triangle \mathrm{dFRC}$ at those PEEP values for each patient

- Assess error between calculated and measured $\Delta$ dFRC values.

These results test a more general approach. Performance is assessed by trend correlation coefficient (R), where.

This approach is unique from the work of Sundaresan et al (2011) in that this prior work required multiple PEEP values to estimate dFRC, which was not practical clinically.

\section{RESULTS}

Table 2 shows the analytical solution for Median [IQR] $\beta_{1}$ for each patient and PEEP and overall cohort results. Figure 3 shows the variation of median $\beta_{1}$ values with respect to PEEP. Figure 4 shows the trend between estimated and measured $\triangle \mathrm{dFRC}$ values with a $\mathrm{R}^{2}=0.862$ using this population constant. Figure 5 shows the general trend observed between the re - estimated and measured $\triangle \mathrm{dFRC}$ using interpolated $\beta_{1}$ values with $\mathrm{R}^{2}=0.850$. Finally, Table 3 shows the percentage error (Median [IQR]) between measured and estimated $\triangle \mathrm{dFRC}$ values. 
Table 2: $\beta_{1}$ values calculated for each patient based on the representative breath chosen, where $4 \_2$ and $6 \_2$ are second trial on the same patient 3 and 8 days later respectively

\begin{tabular}{|c|c|c|c|c|c|c|}
\hline $\begin{array}{l}\text { PEEP } \\
{\left[\mathrm{cmH}_{2} 0\right] 3}\end{array}$ & 5 & 10 & 15 & 20 & 25 & 30 \\
\hline \multicolumn{7}{|l|}{ Patient } \\
\hline 1 & 0.0058 & 0.0160 & 0.0317 & 0.0513 & 0.0679 & \\
\hline 2 & 0.0083 & 0.0209 & 0.0338 & 0.0480 & & \\
\hline 3 & 0.0085 & 0.0231 & 0.0385 & 0.0546 & 0.0678 & \\
\hline 4 & 0.0013 & 0.0070 & 0.0214 & 0.0352 & 0.0442 & 0.0542 \\
\hline 5 & 0.0029 & 0.0051 & 0.0130 & 0.0307 & 0.0545 & \\
\hline $5 \_2$ & & 0.0059 & 0.0190 & 0.0355 & 0.0512 & \\
\hline 6 & 0.0105 & 0.0235 & 0.0399 & 0.0548 & 0.0657 & \\
\hline $6 \_2$ & 0.0132 & 0.0303 & 0.0456 & 0.0589 & & \\
\hline 7 & 0.0189 & 0.0387 & 0.0484 & & & \\
\hline 8 & 0.0098 & 0.0233 & 0.0385 & 0.0545 & 0.0733 & 0.0892 \\
\hline 9 & 0.0057 & 0.0076 & 0.0195 & 0.0322 & 0.0454 & 0.0606 \\
\hline Median & 0.0084 & 0.0209 & 0.0328 & 0.0480 & 0.0545 & 0.0606 \\
\hline IQR & $0.0057,0.0105$ & $0.0072,0.0235$ & $0.0200,0.0396$ & $0.0352,0.0546$ & $0.0483,0.0678$ & $0.0558,0.0821$ \\
\hline
\end{tabular}

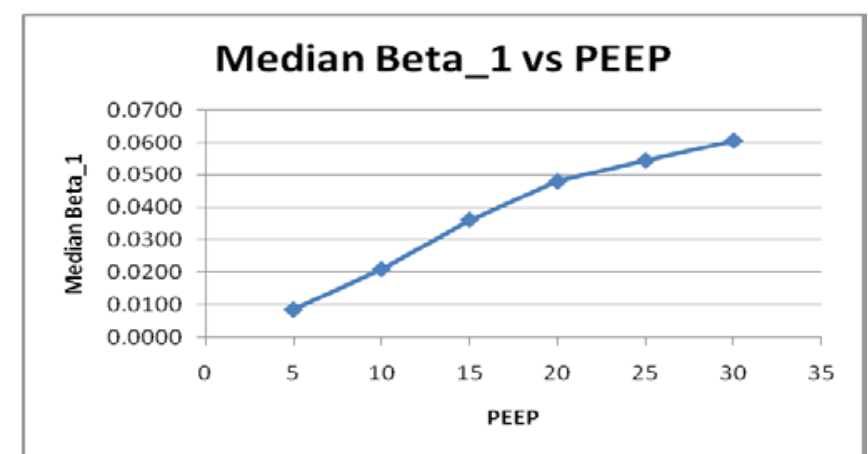

Fig. 3. Median $\beta_{1}$ vs PEEP. The median $\beta_{1}$ here is calculated based on the lung compliance of a representative PV loop.

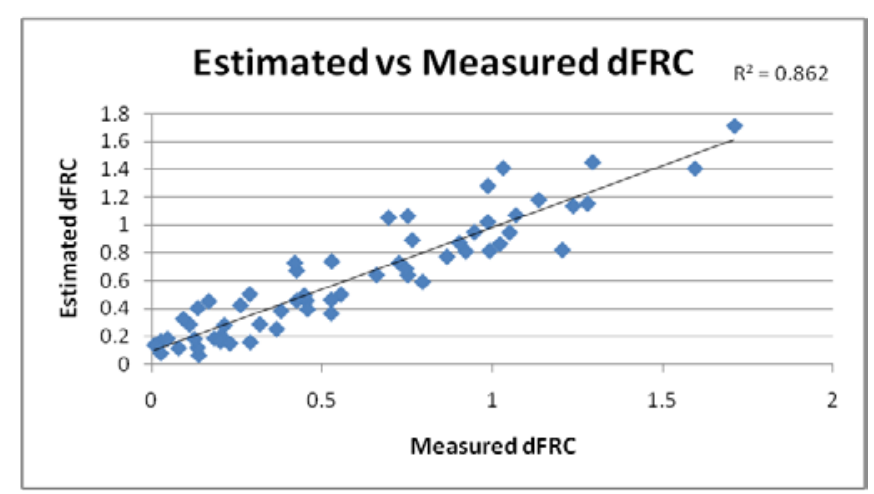

Fig. 4. Estimated vs measured $\triangle \mathrm{dFRC}$ values using exact $\beta_{1}$ values from the cohort at each PEEP.

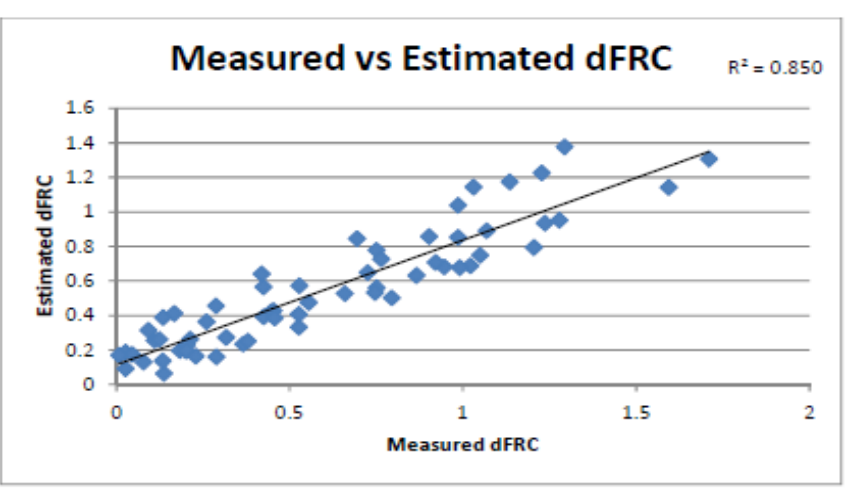

Fig. 5. Measured vs Estimated $\triangle \mathrm{dFRC}$ values using interpolated $\beta_{1}$ values.

\section{DISCUSSION}

The model was developed to estimate $\triangle \mathrm{dFRC}$ as a function of PEEP and tidal volume using only readily available PV data and to do so at a single PEEP setting to avoid interrupting $\mathrm{MV}$ treatment. It estimates $\triangle \mathrm{dFRC}$ using the compliance observed by applying the stress-strain approach proposed by Chiumello et al (Chiumello et al., 2008). Overall, dFRC offers important information on the status of the lung and its recruitability, and using such a non-invasive approach it can be tracked continuously as it changes with the evolution of disease using the proposed method.

It was also observed in Figure 3 and Table 2 that median $\beta_{1}$ values change almost linearly with respect to PEEP at each PEEP level. This linear trend allows the linear interpolation of median $\beta_{1}$ values at intermediate PEEP levels. Thus, to generalise this approach $a b$ value is not needed at every possible PEEP. There was almost no loss in fidelity or correlation as observed comparing the $\mathrm{R}^{2}$ values for the results in Figures 4 and 5. 
Table 3: Percentage error between the observed and measured dFRC values

\begin{tabular}{|c|c|c|c|c|c|c|}
\hline PEEP [cmH $\left.{ }_{2} 0\right]$ & 5 & 10 & 15 & 20 & 25 & 30 \\
\hline \multicolumn{7}{|l|}{ Patient } \\
\hline 1 & 44.18 & 30.65 & 14.00 & 6.42 & 19.75 & \\
\hline 2 & 0.94 & 0.00 & 6.87 & 1.12 & 0.00 & \\
\hline 3 & 0.93 & 9.50 & 6.17 & 12.15 & 19.7 & \\
\hline 4 & 527.41 & 198.63 & 68.62 & 36.18 & 23.22 & 11.78 \\
\hline 5 & 192.84 & 311.93 & 177.03 & 56.35 & 0.00 & \\
\hline $5 \_2$ & & 253.81 & 89.93 & 35.28 & 6.38 & \\
\hline 6 & 19.93 & 11.16 & 9.42 & 12.40 & 17.05 & \\
\hline $6 \_2$ & 36.40 & 31.12 & 20.80 & 18.48 & & \\
\hline 7 & 55.39 & 46.06 & 25.27 & & & \\
\hline 8 & 14.20 & 10.32 & 6.04 & 11.92 & 25.68 & 32.74 \\
\hline 9 & 47.96 & 174.96 & 85.20 & 48.96 & 19.96 & 0.06 \\
\hline Median & 40.29 & 31.12 & 20.8 & 15.44 & 19.7 & 11.78 \\
\hline IQR & $14.20,55.39$ & $10.53,192.71$ & $7.51,81.06$ & $11.92,36.18$ & $4.79,20.76$ & $2.99,27.5$ \\
\hline
\end{tabular}

Sundaresan et al (Sundaresan et al., 2011) reported $\mathrm{R}^{2}=$ 0.946 over the entire cohort. However, a significant drawback in the model proposed by Sundaresan et al was that it could not be used for continuously tracking dFRC since it requires PV data for at least 2 PEEP levels. Its application in real-time $\mathrm{dFRC}$ measurement is thus limited without interrupting MV treatment and consuming clinical time.

The new model proposed here utilises readily available clinical data from a single breath. It can thus be automated and does not interrupt MV therapy nor require any special clinical intervention. These advantages allow continuous tracking of $\triangle \mathrm{dFRC}$ and thus of $\mathrm{dFRC}$ if FRC is measured. The compromise in accuracy is acceptable to maintain this advantage.

However, it must be noted that the method proposed has some limitations in its predictive capability. In some cases, the percentage error observed between the estimated and measured $\triangle \mathrm{dFRC}$ values was high, up to almost $500 \%$ in one of the patients studied. Larger errors were primarily found at very low PEEP levels, below Auto - PEEP (Sunderesan, 2010). Such low levels are generally not observed in clinical settings. Equally, Auto-PEEP can be detected directly from PV loop responses. Thus, this issue is manageable. In particular, ignoring low PEEP values less than known AutoPEEP levels (Sundaresan et al 2011b) yields maximum errors of only XXXX\%.

In general, larger estimation errors can limit the use of this or any similar method for estimating the recruitment potential. However, median percentage errors in all cases were $10-30 \%$ and generally lower as PEEP rose above Auto-PEEP. Equally, the trends were still valid.

In particular, estimated $\triangle \mathrm{dFRC}$ follows a similar trend with respect to PEEP as the actual $\triangle \mathrm{dFRC}$. Hence in spite of higher error values in limited cases, the model is still capable of predicting the trend in $\triangle \mathrm{dFRC}$ with respect to PEEP. This particular outcome is shown in Figure 6, which shows the measured and estimated $\triangle \mathrm{dFRC}$ values for Patient 5 where percentage errors observed were high at low PEEP values due to an Auto-PEEP of XXX cmH2O. It is clear that while there is bias, the trend is quite accurate, which would allow effective clinical assessment and use.

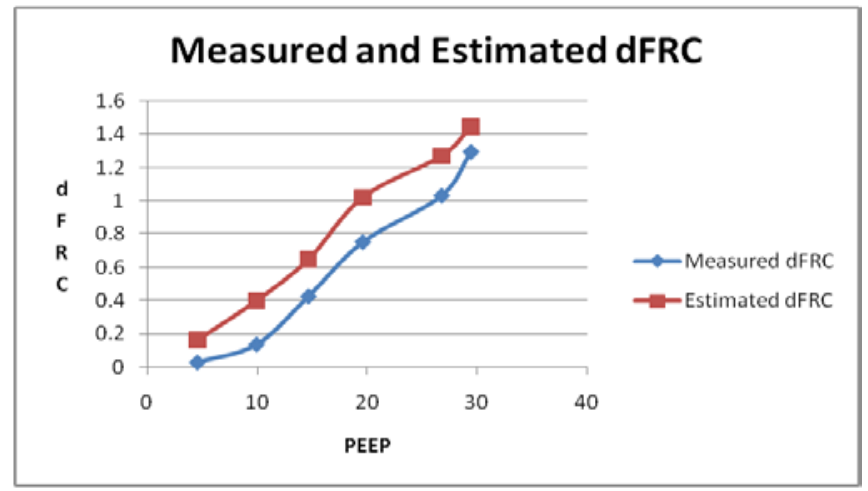

Fig. 6. Measured and Estimated dFRC for patient 5

Another limitation of this model is the assumption that the specific lung Elastance, $\mathrm{E}_{\mathrm{Lspec}}$ and $\alpha$ remain constant. It should be noted that these values remain constant over the linear portion of the PV curve. The compliance calculated is expected to follow the linear portion of the PV curve, but this assumption may not always be true, as seen at very low PEEP values and those below any Auto-PEEP or, potentially, at very high clinically unrealistic PEEP values where obvious lung over distension occurs. Thus, the overall approach is feasible and accurate for the range of clinically acceptable PEEP values seen in application.

\section{CONCLUSIONS}

A novel method of estimating dFRC for a given mechanically ventilated patient is presented, based on a stress-strain approach. The method presented allows real-time assessment using data from only a single PEEP level. Thus, there needs to be no interruption to MV therapy or any added clinical effort to obtain this value - a completely non-invasive, model-based approach. The accuracy of the resulting values is only slightly reduced from methods that require several PEEP levels and significant, invasive clinical intervention. The trends over time and PEEP are equally accurate ensuring that there is no loss in clinical applicability and value. 


\section{REFERENCES}

Bersten, A. D. (1998). Measurement of overinflation by multiple linear regression analysis in patients with acute lung injury. Eur. Respir.J, volume 12, 526-532.

Bersten, A. D., Edibam, C., Hunt, T. and Moran, J. (2002). Incidence and Mortality of Acute Lung Injury and the Acute Respiratory Distress Syndrome in Three Australian States. Am. J. Respir. Crit. Care Med., volume 165, 443448.

Chiumello, D., Carlesso, E., Cadringher, P., Caironi, P., Valenza, F., Polli, F., Tallarini, F., Cozzi, P., Cressoni, M., Colombo, A., Marini, J. J. and Gattinoni, L. (2008). Lung Stress and Strain during Mechanical Ventilation for Acute Respiratory Distress Syndrome. Am J Respir Crit Care Med, volume 178, 346-355.

Gattinoni, L., Chiumello, D., Carlesso, E. and Valenza, F. (2004). Bench-to-bedside review: Chest wall elastance in acute lung injury/acute respiratory distress syndrome patients. Critical Care, volume 8, 350:355.

Gattinoni, L. and Pesenti, A. (2005). The concept of "baby lung”. Intensive Care Medicine, volume 31, 776-784.

Ge Healthcare. 2006. The INview Suite: SpiroDynamics and FRC INview. .

Heinze, H., Schaaf, B., Grefer, J., Klotz, K. and Eichler, W. (2007). The Accuracy of the Oxygen Washout Technique for Functional Residual Capacity Assessment During Spontaneous Breathing. Anesth. Analg., volume 104, 598604.

Luhr, O. R., Antonsen, K., Karlsson, M., Aardal, S., Thorsteinsson, A., Frostell, C. G. and Bonde, J. a. N. (1999). Incidence and Mortality after Acute Respiratory Failure and Acute Respiratory Distress Syndrome in Sweden, Denmark, and Iceland. the arf study group. Am. J. Respir. Crit. Care Med., volume 159, 1849-1861.

Malbouisson, L. M., Muller, J. C., Contantin, J. M., Lu, Q., Puybasset, L., Rouby, J. J. and Group, A. T. C. S. S. A. (2001). Computed Tomography Assessment of Positive End-expiratory Pressure-induced Alveolar Recruitment in Patients with Acute Respiratory Distress Syndrome. American Journal of Respiratory and Critical Care Medicine, volume 163, 1444-1450.

Manzano, F., Yuste, E., Colmenero, M., Aranda, A., GarcíaHorcajadas, A., Rivera, R. and Fernández-Mondéjar, E. (2005). Incidence of acute respiratory distress syndrome and its relation to age. Journal of Critical Care, volume 20, 274-280.

Olegard, C., Sondergaard, S., Houltz, E., Lundin, S. and Stenqvist, O. (2005). Estimation of Functional Residual Capacity at the Bedside Using Standard Monitoring Equipment: A Modified Nitrogen Washout/Washin Technique Requiring a Small Change of the Inspired Oxygen Fraction. Anesth. Analg., volume 101, 206-12.

Suchyta, M. R., Clemmer, T. P., Elliott, C. G., Orme, J. F., Morris, A. H., Jacobson, J. and Menlove, R. (1997). Increased Mortality of Older Patients With Acute Respiratory Distress Syndrome. Chest, volume 111, 1334 1339.

Sundaresan, A., Chase, G., Hann, C. and Shaw, G. (2011). Dynamic functional residual capacity can be estimated using a stress-strain approach. Computer Methods and Programs in Medicine, volume 101, 135-143.

Sunderesan, A. 2010. Applications of Model-Based Lung Mechanics in the Intensive Care Unit. Doctor of Philosophy, The University of Canterbury.

Ware, L. B. and Matthay, M. A. (2000). The acute respiratory distress syndrome. N Engl J Med, volume 342, 1334-49.

Weismann, D., Reißmann, H., Maisch, S., Füllekrug, B. and Schulte, J. (2006). Monitoring of Functional Residual Capacity by an Oxygen Washin/Washout; Technical Description and Evaluation. Journal of Clinical Monitoring and Computing, volume 20, 251-260.

Yuta, T. 2007. Minimal Model of Lung Mechanics for Optimising Ventilator Terapy in Critical Care. Doctor of Philosophy The University of Canterbury. 